\title{
Effect of substitutional defects on resonant tunneling diodes based on armchair graphene and boron nitride nanoribbons lateral heterojunctions
}

\author{
Majid Sanaeepur ${ }^{1,2}$
}

\author{
Full Research Paper \\ Address: \\ ${ }^{1}$ Department of Electrical Engineering, Faculty of Engineering, Arak \\ University, Arak, 3815688349, Iran and ${ }^{2}$ Institute of Nanosciences \\ and Nanotechnology, Arak University, Arak, Iran \\ Email: \\ Majid Sanaeepur - m-sanaeepur@araku.ac.ir \\ Keywords: \\ AGNR/ABNNR heterojunction; armchair boron nitride nanoribbon \\ (ABNNR); armchair graphene nanoribbon (AGNR); negative \\ differential resistance (NDR); nonequilibrium Green's function \\ (NEGF); resonant tunneling diode (RTD); substitutional defects \\ Beilstein J. Nanotechnol. 2020, 11, 688-694. \\ doi:10.3762/bjnano.11.56 \\ Received: 11 November 2019 \\ Accepted: 14 April 2020 \\ Published: 24 April 2020 \\ Associate Editor: E. Meyer \\ (C) 2020 Sanaeepur; licensee Beilstein-Institut. \\ License and terms: see end of document.
}

Open Access

\begin{abstract}
A nanometer-scaled resonant tunneling diode based on lateral heterojunctions of armchair graphene and boron nitride nanoribbons, exhibiting negative differential resistance is proposed. Low-bandgap armchair graphene nanoribbons and high-bandgap armchair boron nitride nanoribbons are used to design the well and the barrier region, respectively. The effect of all possible substitutional defects (including $\mathrm{B}_{\mathrm{C}}, \mathrm{N}_{\mathrm{C}}, \mathrm{C}_{\mathrm{B}}$, and $\mathrm{C}_{\mathrm{N}}$ ) at the interface of graphene and boron nitride nanoribbons on the negative differential resistance behavior of the proposed resonant tunneling diode is investigated. Transport simulations are carried out in the framework of tight-binding Hamiltonians and non-equilibrium Green's functions. The results show that a single substitutional defect at the interface of armchair graphene and boron nitride nanoribbons can dramatically affect the negative differential resistance behavior depending on its type and location in the structure.
\end{abstract}

\section{Introduction}

2D materials have gained tremendous research interest due to the unique properties that result from their atomic-scale thickness [1-5]. These materials, which include graphene, hexagonal boron nitride, and the large family of transition metal dichalcogenides, have electronic structures exhibiting metallic, semiconducting, and insulating properties. Novel electronic devices have been realized by heterostructures based on vertical stacking or lateral stitching of 2D materials with different elec- tronic properties [6]. Lateral graphene/hexagonal boron nitride $(\mathrm{Gr} / \mathrm{hBN})$ heterostructures, due to very low lattice mismatch between graphene and hBN, are most suitable as platforms for fully two-dimensional nanoelectronic devices [7-11].

Resonant tunneling diodes (RTDs) are among various electronic devices realized on the platform of $2 \mathrm{D} \mathrm{Gr} / \mathrm{hBN}$ heterostructures [12-16]. RTDs exhibit negative differential resis- 
tance (NDR) and have a wide range of applications including ultra-fast switching devices, oscillators, frequency multipliers, one-transistor static memories and multi-valued memory circuits [12,17-20].

In a RTD, a material with low bandgap energy is sandwiched between two materials with larger bandgaps, i.e., a quantum well between two potential barriers, forming a so-called doublebarrier quantum well structure. In the well, the energy of the electrons is quantized due to the quantum confinement of their wave function. Incident electrons with energies equal to the quantized levels of the well pass through the barriers with rather high transmission probabilities. Electrons with other energies have an extremely small chance of passing through. This causes RTDs to exhibit NDR in their current-voltage characteristic.

Conventionally, RTDs are made by vertical stacking of bulk semiconductor materials with different bandgap energies to form a planar 3D structure in which the direction of carrier transport is perpendicular to the interface of stacked materials [21-23]. In recent years, a few RTD structures based on 2D materials have been proposed [24-26]. In such RTDs the bandgap difference needed for normal operation is created by juxtaposing graphene nanoribbons (GNRs) with different widths (utilizing the inverse relation between GNR width and bandgap energy) or by periodically arranging graphene (the well) and boron nitride regions (the barriers). While the performance of conventional RTDs based on bulk semiconductors is degraded by dislocations and lattice mismatch at the interface of different bandgap materials, the RTDs based on heterojunctions between armchair graphene nanoribbons (AGNRs) and armchair boron nitride nanoribbons (ABNNRs) have shown superior performance because of the very low lattice mismatch between graphene and hBN [3]. However, inevitable interfacial defects located at the interface of $\mathrm{Gr} / \mathrm{hBN}$ heterojunctions, including point defects (single vacancies and substitutional defects) and topological defects can alter the electronic properties of $\mathrm{Gr} / \mathrm{hBN}$ heterostructures and, consequently, the performance of RTDs based on $\mathrm{Gr} / \mathrm{hBN}$ heterojunctions [26-33]. Formation energy calculations have revealed that point defects occur preferentially at the interfaces of graphene and $\mathrm{hBN}$ domains rather than in the middle of these domains, and that substitutional defects are dominant [30]. Therefore, a reliable and accurate investigation of the electronic behavior in devices based on $\mathrm{Gr} / \mathrm{hBN}$ heterostructures must consider the effect of such defects.

In this work, a nanometer-scaled RTD based on lateral AGNR/ ABNNR heterojunctions is proposed and the effect of all possible types of substitutional defects at the interface of
AGNR/ABNNR heterojunctions on the electronic behavior of the proposed RTD is investigated.

The effect of substitutional defects on the electronic transport properties of zigzag graphene nanoribbons symmetrically decorated with $\mathrm{BN}$ is described in [34]. However, it considers the interfaces of the $\mathrm{Gr} / \mathrm{hBN}$ regions are parallel to the transport direction so that there are no bandgap variations in the transport direction. Since the electron transport is in zigzag direction, spin-polarized transport calculations are utilized. Instead, in the study presented here, the electron transport is in armchair direction, which is not spin-polarized. Therefore, nonequilibrium Green's functions with tight-binding Hamiltonians (without considering spin degree of freedom) are utilized for electronic transport calculations. Furthermore, in the proposed RTD there are four GNR/BNNR heterojunctions perpendicular to the transport direction (Figure 1), which are required to construct the double-barrier quantum well structure.

\section{Calculation Method}

Figure 1a schematically shows the structure of the proposed RTD. An AGNR of 19 carbon atoms in width (19-AGNR) and four hexagonal carbon rings in length $\left(1.7 \times 1.6 \mathrm{~nm}^{2}\right)$ is sandwiched between two ABNNRs of the same width but of only one hexagonal $\mathrm{hBN}$ ring in length $\left(1.7 \times 0.42 \mathrm{~nm}^{2}\right)$. Two spacer regions are considered at both sides of the structure to exclude outward interfacial defects from the contact regions. The whole structure is assumed to be connected to semi-infinite AGNR contacts at both sides. The energy band edge diagram of the proposed RTD along with the quantized energy level of the

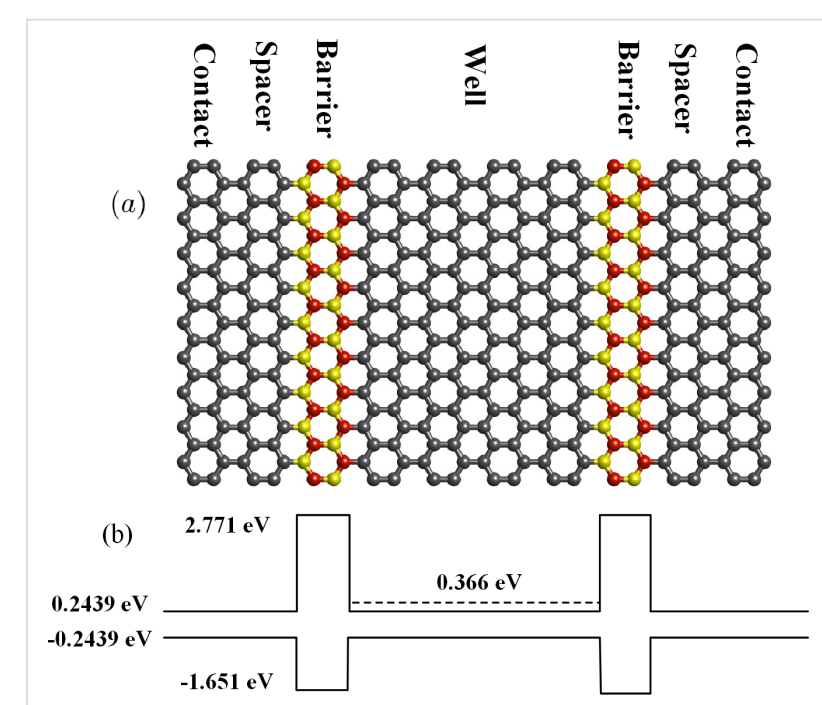

Figure 1: (a) Schematic representation of the proposed RTD. Carbon, boron and nitrogen atoms, are depicted in grey, red and yellow, respectively. The contacts are assumed to be heavily doped semi-infinite AGNRs. (b) Energy diagram of the proposed RTD (solid lines) and resonant energy of the well (dashed line). 
well are shown in Figure 1b. Due to small lattice mismatch between graphene and hBN the edge bond relaxation correction for carbon atoms at the interface of $\mathrm{C}$ and $\mathrm{hBN}$ domains could be ignored [35]. Therefore, the electronic structure of the proposed RTD could be modeled via a tight-binding Hamiltonian, $H_{\mathrm{D}}$, including first nearest-neighbor interactions [36]. The hopping energies between $\mathrm{C}, \mathrm{B}$ and $\mathrm{N}$ atoms and the on-site energies of $\mathrm{B}$ and $\mathrm{N}$ atoms are listed in Table 1 [37].

Table 1: Tight-binding parameters for the CBN composite structure ${ }^{a}$

\begin{tabular}{lllllll}
$\varepsilon_{\mathrm{B}}$ & $\varepsilon_{\mathrm{C}}$ & $\varepsilon_{\mathrm{N}}$ & $t_{\mathrm{CC}}$ & $t_{\mathrm{CB}}$ & $t_{\mathrm{CN}}$ & $t_{\mathrm{BN}}$ \\
\hline 2.76 & 0.00 & -1.64 & 2.65 & 2.25 & 1.70 & 2.40 \\
\hline
\end{tabular}

${ }^{\mathrm{a}} \varepsilon$ and $t$ denote the on-site and hopping energies, respectively (both in $\mathrm{eV}$ ).

In nonequilibrium Green's functions the retarded Green's function of the device (scattering region) is written as [38]:

$$
G^{r}(E)=\left[(E+i \eta) I-H_{\mathrm{D}}-\Sigma(E)\right]^{-1},
$$

where $\eta$ is an infinitesimal positive number and the nonHermitian self-energy matrix, $\Sigma(E)=\Sigma_{1}(E)+\Sigma_{2}(E)$, represents the escape rate of electrons from the device into the semi-infinite contacts. The self-energy matrices are calculated through a highly convergent recursive method [39]. Then the transmission as a function of the energy is obtained via [39]:

$$
T(E)=\operatorname{Tr}\left[\Gamma_{2}(E) G^{\mathrm{r}}(E) \Gamma_{1}(E) G^{\mathrm{a}}(E)\right]
$$

in which $G^{\mathrm{a}}(E)=\left(G^{\mathrm{r}}(E)\right)^{\dagger}$ is the advanced Green's function and $\Gamma_{j}(j=1,2)$, represent the level broadening due to the coupling between device and contacts:

$$
\Gamma_{\mathrm{j}}(E)=i\left[\Sigma_{\mathrm{j}}(E)+\Sigma_{\mathrm{j}}^{\dagger}(E)\right], \quad i=\sqrt{-1}
$$

Finally, the current through the device is calculated via [38]:

$$
I=\frac{2 q}{h} \int_{-\infty}^{\infty} \mathrm{d} E T(E)\left[f_{1}(E)-f_{2}(E-q V)\right]
$$

in which $q$ is the electron charge, $f(E)$ is the Fermi-Dirac distribution function at the contacts and $V$ is the bias voltage. The Fermi level at both contacts is assumed to be $0.03 \mathrm{eV}$ above the conduction band edge [40]. Room temperature $(300 \mathrm{~K})$ is considered in all simulations.

\section{Results and Discussion}

Substitutional carbon atoms in boron or nitrogen sublattices $\left(\mathrm{C}_{\mathrm{B}}\right.$ and $\left.\mathrm{C}_{\mathrm{N}}\right)$ as well as boron or nitrogen atoms on carbon sites $\left(B_{C}\right.$ and $\mathrm{N}_{C}$ ) are considered (Figure 2). Each defect could occur inside the well or in the contact regions. Since the typical defect concentration for real samples is one defect per ca. $10 \AA$, one defect of each type is considered at the $\mathrm{Gr} / \mathrm{hBN}$ heterojunctions [41].

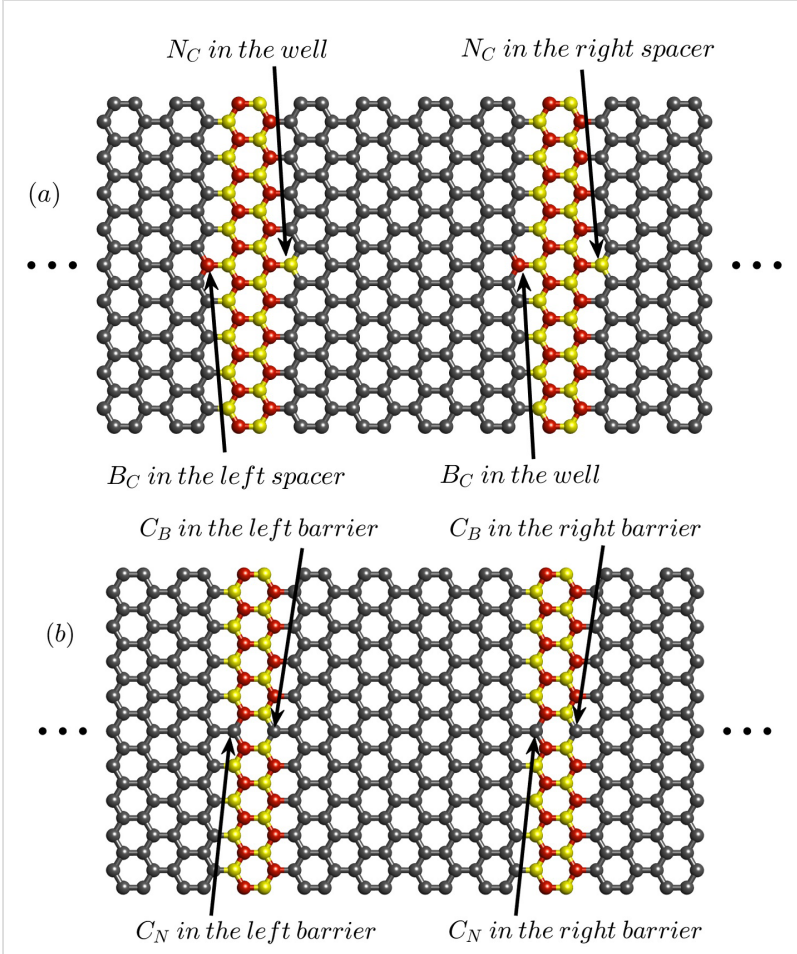

Figure 2: All possible substitutional defects at the interfaces of RTD based on AGNR/ABNNR heterojunctions. Carbon, boron and nitrogen atoms are depicted in grey, red and yellow, respectively.

Figure 3 shows the effect of substitutional defects on the energy bandstructure of 19-AGNR and 19-ABNNR. Both $\mathrm{B}_{\mathrm{C}}$ and $\mathrm{N}_{\mathrm{C}}$ defects increase the bandgap of AGNR (Figure 3b,c) due to breaking the symmetry between two graphene sublattices. A $\mathrm{B}_{\mathrm{C}}$ defect, due to the p-type character, shifts the conduction band edge toward higher energies, while an $\mathrm{N}_{\mathrm{C}}$ defect, due to the n-type character, shifts it toward lower energies. In contrast, both $C_{B}$ and $C_{N}$ defects decrease the bandgap of ABNNR. However, a $C_{B}$ defect in $A B N N R$, due to the n-type character, shifts the conduction band edge toward lower energies while the $\mathrm{C}_{\mathrm{N}}$ defect, due to the p-type character, shifts it toward higher energies (Figure 3e,f).

Figure $4 a$ depicts the effect of $C_{B}$ and $N_{C}$ substitutional defects at the interface between the left ABNNR barrier and the well on the current-voltage characteristic of the proposed RTD. Both 

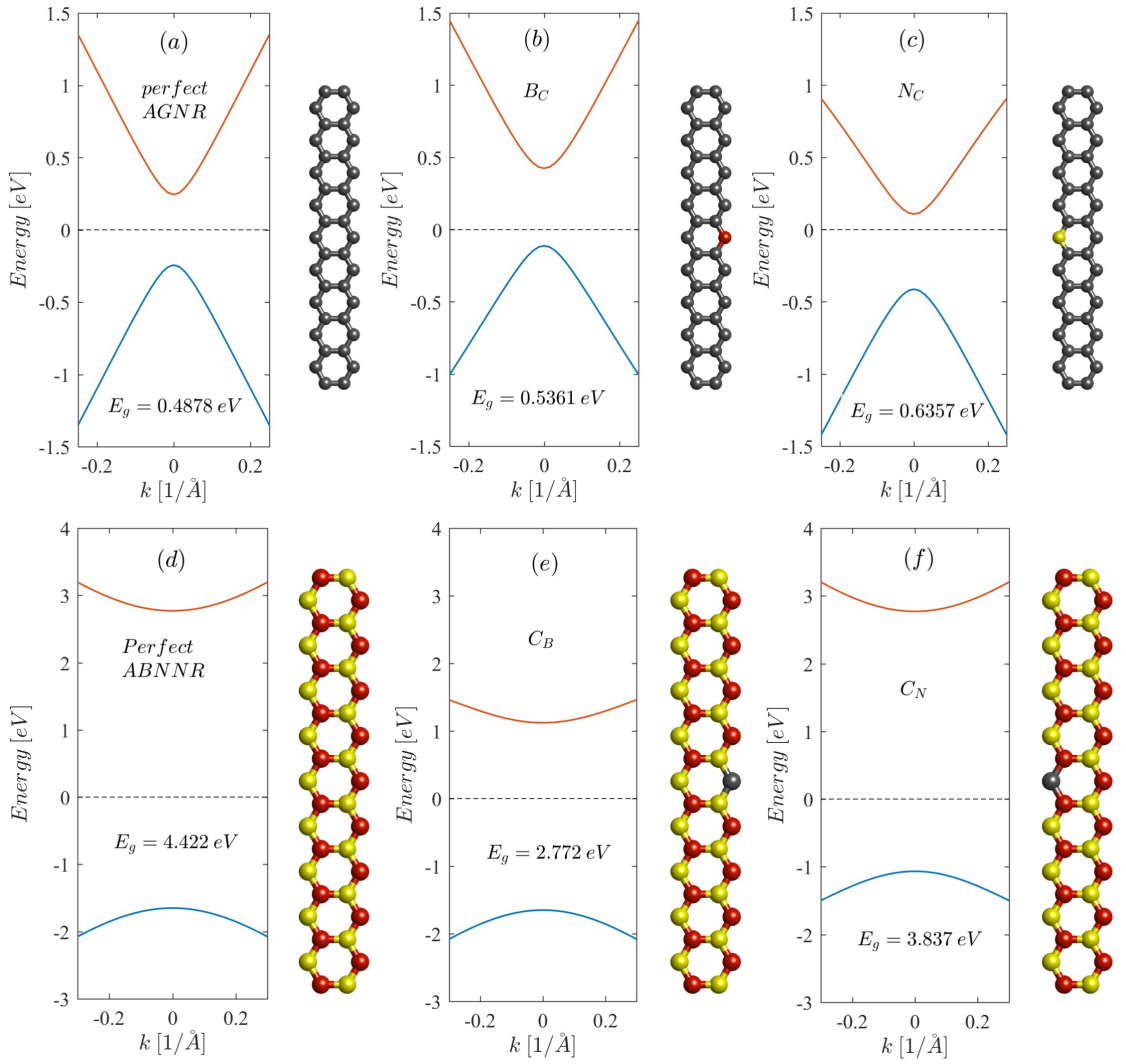

Figure 3: Effect of substitutional defects on the energy bandstructure of 19-AGNR and 19-ABNNR. Carbon, boron and nitrogen atoms are depicted in grey, red and yellow, respectively.
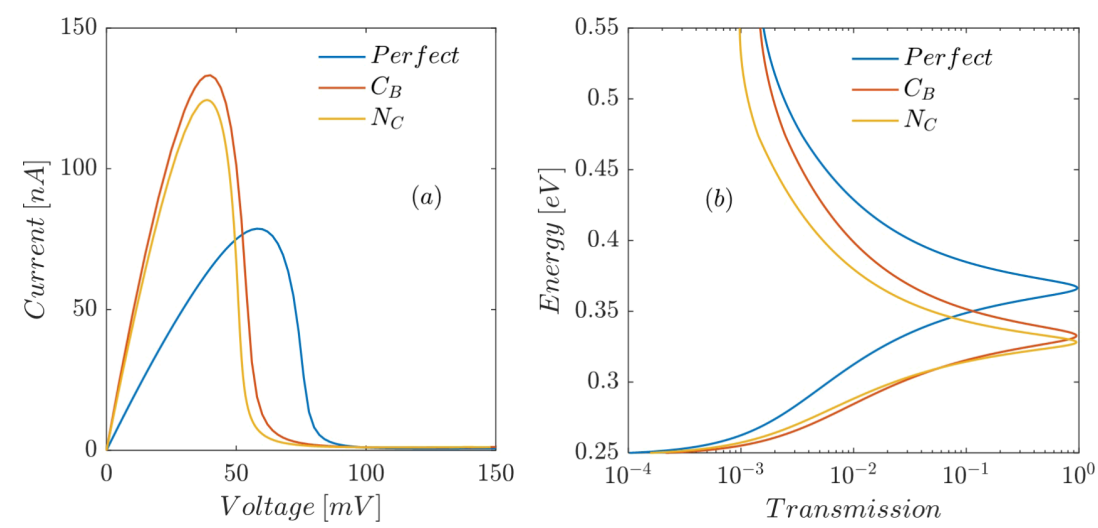

Figure 4: Effect of $\mathrm{C}_{\mathrm{B}}$ and $\mathrm{N}_{\mathrm{C}}$ defects at the interface between the left $A B N N R$ barrier and the well on (a) the current-voltage characteristic and (b) the transmission trough the proposed RTD as a function of the electron energy.

defects lower the on-site energy at the place of the substituted atom due to the n-type character, which shifts all energy levels including the conduction band edge toward lower energies. The $\mathrm{N}_{\mathrm{C}}$ defect inside the well also lowers the resonant energies of the well. Therefore, the transmission peaks move downward (Figure $4 \mathrm{~b}$ ), which causes the peak current $\left(I_{\mathrm{p}}\right)$ to increase and the peak voltage $\left(V_{\mathrm{p}}\right)$ to decrease (Figure $\left.4 \mathrm{a}\right) . \mathrm{A} \mathrm{C}_{\mathrm{B}}$ defect in the left barrier lowers the conduction band edge (Figure 3e). This also, by reducing the height of the left energy barrier, lowers the resonant energies of the well, which in turn moves the transmission peaks downward (Figure 4b). Lower transmission peaks translate to decreased $V_{\mathrm{p}}$ and increased $I_{\mathrm{p}}$ (Figure $4 \mathrm{a}$ ). 
Both $B_{C}$ and $C_{N}$ substitutional defects have p-type character (adding an extra hole to the system) because the added atom has one electron less than the removed atom. Therefore, $\mathrm{a}_{\mathrm{C}}$ defect in the well shifts all allowed energy levels (including discrete resonant energies) upward (Figure $3 \mathrm{~b}$ ), which causes $V_{\mathrm{p}}$ to increase and $I_{\mathrm{p}}$ to decrease (Figure $5 \mathrm{a}$ ). A $\mathrm{C}_{\mathrm{N}}$ defect at the interface between the right barrier and the well (inside the barrier) also moves the conduction band edge upward, which in turn shifts the resonant energies of the well upward causing $V_{\mathrm{p}}$ to increase and $I_{\mathrm{p}}$ to decrease (Figure 5a).

$\mathrm{C}_{\mathrm{B}}$ and $\mathrm{N}_{\mathrm{C}}$ defects may occur at the interface between the right barrier and the right spacer (see Figure 2). Because these substitutional defects are outside the well region they have no effect on the resonant energies of the well (Figure 6b). Therefore, in both cases $V_{\mathrm{p}}$ remains unchanged as illustrated in Figure 6a. Nevertheless, a $C_{B}$ defect in the right barrier lowers the conduction band edge and consequently the barrier height. Therefore, the transmission probabilities over the right barrier at all ener- gies are increased compared to a defect-free RTD (red curve in Figure $6 \mathrm{~b}$ ). The $\mathrm{N}_{\mathrm{C}}$ defect in the right spacer also lowers the conduction band edge. This creates a local quantum well in the right spacer region, which, by localizing electron wave functions, reduces the transmission coefficients (yellow curve in Figure $6 \mathrm{~b}$ ). This causes $I_{\mathrm{p}}$ to decrease compared to a defect-free structure.

Figure 7 compares the current-voltage characteristic of RTDs including $\mathrm{B}_{\mathrm{C}}$ and $\mathrm{C}_{\mathrm{N}}$ defects at the interface between the left barrier and the left spacer with that of a defect-free RTD. If there is no defect in the left spacer, by increasing the bias voltage a quantum well is formed in this region, which localizes incident electron waves. However, a $\mathrm{B}_{\mathrm{C}}$ defect in the left spacer region shifts the conduction band edge upward. Thus, a potential barrier with a very small height (with respect to the ABNNR potential barrier) is formed. Although by increasing the bias voltage the height of this potential barrier is lowered, a quantum well is never formed in the spacer region in this case
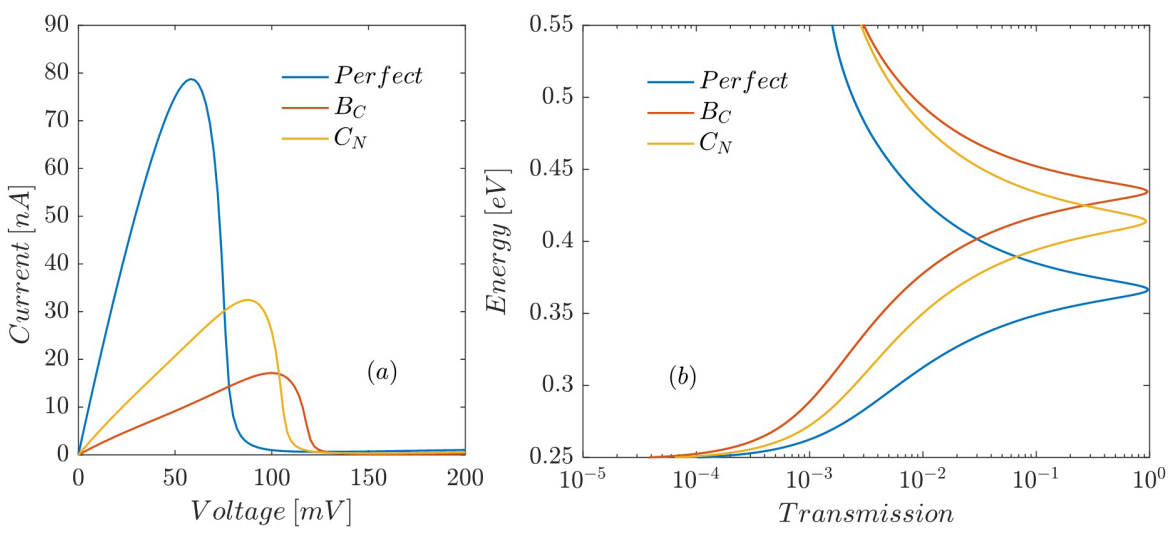

Figure 5: Effect of $\mathrm{B}_{\mathrm{C}}$ and $\mathrm{C}_{\mathrm{N}}$ defects at the interface between the right barrier and the well on (a) current-voltage characteristic and (b) the transmission trough the proposed RTD as a function of the electron energy.
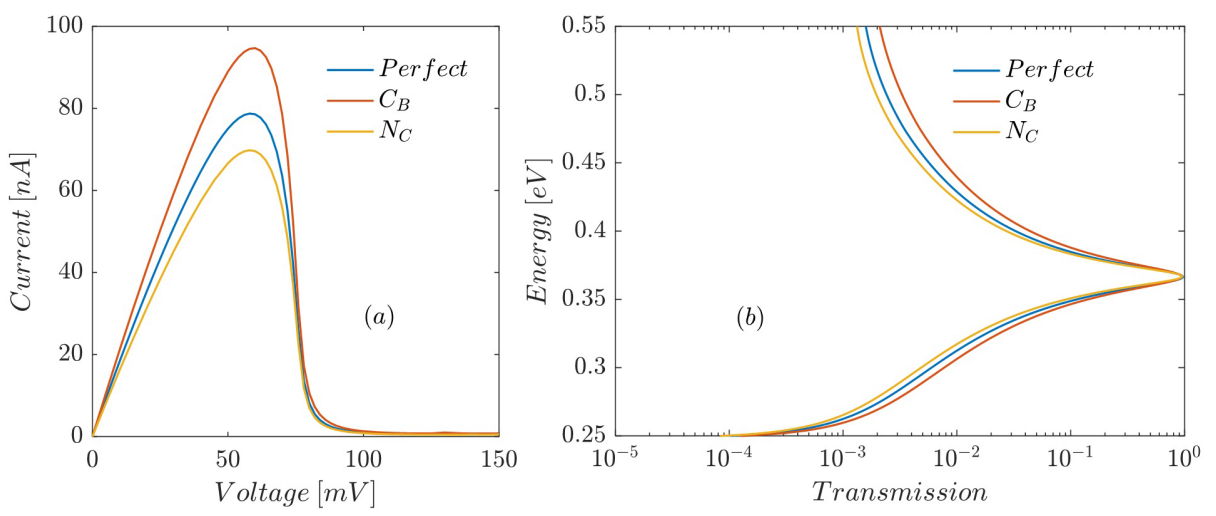

Figure 6: Effect of $\mathrm{C}_{B}$ and $\mathrm{N}_{C}$ defects at the interface between the right barrier and the right spacer on (a) current-voltage characteristic and (b) the transmission trough the proposed RTD as a function of the electron energy. 

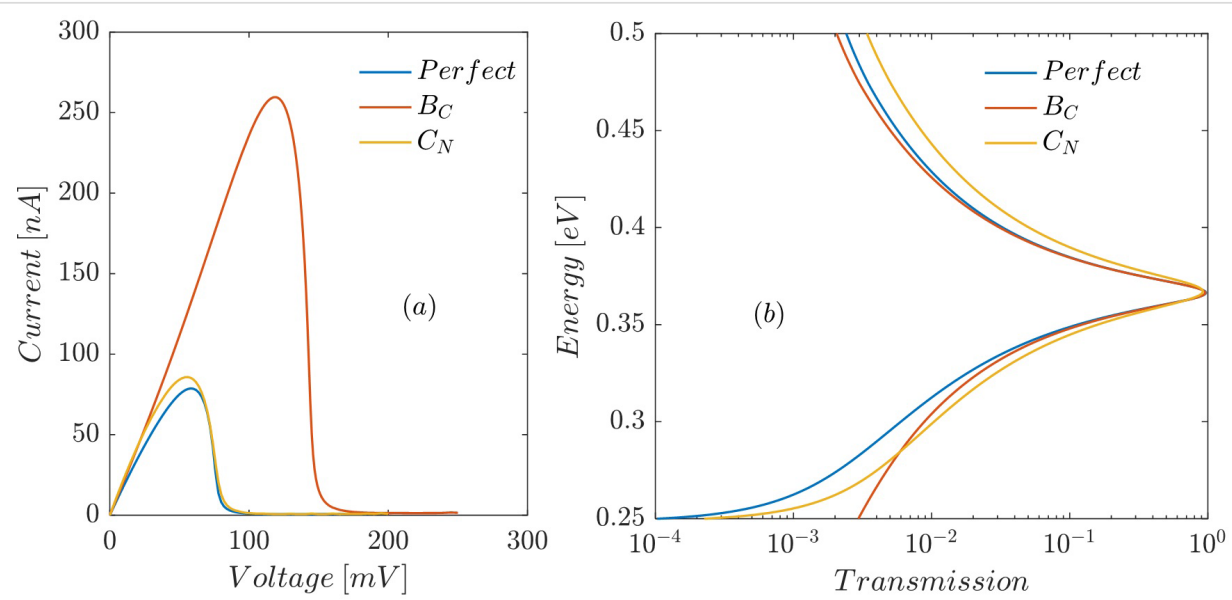

Figure 7: Effect of $B_{C}$ and $C_{N}$ defects at the interface between the left barrier and the left spacer on (a) current-voltage characteristic and (b) the transmission trough the proposed RTD as a function of the electron energy.

(at least up to a bias voltage of $250 \mathrm{mV}$, which is considered in this work). The absence of a local quantum well in the left spacer region (for bias voltages ranging from 0 to $250 \mathrm{mV}$ ) causes the transmission peak to vanish at larger bias voltages with respect to the defect-free structure. Therefore, the peak values of current and voltage are increased with respect to defect-free structure (Figure 7a). A $\mathrm{C}_{\mathrm{N}}$ defect in the left barrier region, besides reducing the bandgap, slightly lowers the conduction band edge (Figure 3f). This has a twofold effect: a small downward shift of the resonant energies of the well and a slight increase in transmission probabilities over the left barrier (Figure $7 \mathrm{~b}$ ), which in turn cause $I_{\mathrm{p}}$ to slightly increase and $V_{\mathrm{p}}$ to slightly decrease, as illustrated by the yellow curve in Figure 7a. Thus, a single substitutional defect can severely alter the NDR behavior of RTDs based on AGNR/ABNNR heterojunctions. This implies that by intentionally introducing such defects in devices based on AGNR/ABNNR heterojunctions, nanoelectronic devices with desired performance characteristics can be designed.

\section{Conclusion}

The effect of substitutional defects on the NDR behavior of a nanometer-scaled RTD based on 2D heterojunctions of AGNR/ ABNNR was investigated. It was shown that a single substitutional defect, depending on its type and position, could severely alter the NDR behavior of the proposed RTD. While a $\mathrm{B}_{\mathrm{C}}$ defect inside the well region decreases $I_{\mathrm{p}}$ and increases $V_{\mathrm{p}}$, it increases both $I_{\mathrm{p}}$ and $V_{\mathrm{p}}$ if located at the interface between left barrier and spacer. An $\mathrm{N}_{\mathrm{C}}$ defect inside the well increases $I_{\mathrm{p}}$ and decreases $V_{\mathrm{p}}$, while it decreases $I_{\mathrm{p}}$ and does not affect $V_{\mathrm{p}}$ if located at the interface between right barrier and spacer. Although a $\mathrm{C}_{\mathrm{N}}$ defect inside the well has the same effect as a $\mathrm{B}_{\mathrm{C}}$ defect, it increases $I_{\mathrm{p}}$ and decreases $V_{\mathrm{p}}$ if located at the interface between left barrier and spacer. $\mathrm{A}_{\mathrm{B}}$ defect inside the well has the same effect as an $\mathrm{N}_{\mathrm{C}}$ defect, while it decreases $I_{\mathrm{p}}$ and does not alter $V_{\mathrm{p}}$ if located at the interface between right barrier and spacer. Substitutional defects can be intentionally incorporated in 2D heterojunctions in a controllable manner. Therefore, since peak current and peak voltage of the proposed RTD depend on the position of the resonant energy of the well, and the resonant energy itself is highly sensitive to the type and position of substitutional defects, the intentional introduction of such defects can be utilized to design nanoscale RTDs with desired NDR characteristic and RTD-based strain or pressure sensors with improved sensitivity.

\section{ORCID ${ }^{\circledR}$ iDs}

Majid Sanaeepur - https://orcid.org/0000-0001-8818-2897

\section{References}

1. Liu, B.; Abbas, A.; Zhou, C. Adv. Electron. Mater. 2017, 3, 1700045. doi:10.1002/aelm.201700045

2. Shim, J.; Park, H.-Y.; Kang, D.-H.; Kim, J.-O.; Jo, S.-H.; Park, Y.; Park, J.-H. Adv. Electron. Mater. 2017, 3, 1600364. doi:10.1002/aelm.201600364

3. Sanaeepur, M.; Jafari, M. R.; Esmaeili, M. IEEE Trans. Electron Devices 2020, 67, 725-729. doi:10.1109/ted.2019.2958421

4. Sanaeepur, M. IEEE Trans. Nanotechnol. 2019, 18, 1181-1187. doi:10.1109/tnano.2019.2945354

5. Sanaeepur, M. IEEE Trans. Electromagn. Compat. 2019, 61, 532-537. doi:10.1109/temc.2018.2830182

6. Li, M.-Y.; Chen, C.-H.; Shi, Y.; Li, L.-J. Mater. Today 2016, 19, 322-335. doi:10.1016/j.mattod.2015.11.003

7. Fiori, G.; Betti, A.; Bruzzone, S.; Iannaccone, G. ACS Nano 2012, 6, 2642-2648. doi:10.1021/nn300019b

8. Sanaeepur, M.; Yazdanpanah Goharrizi, A.; Sharifi, M. J. Beilstein J. Nanotechnol. 2014, 5, 1569-1574. doi:10.3762/bjnano.5.168

9. Goharrizi, A. Y.; Sanaeepur, M.; Sharifi, M. J. Superlattices Microstruct. 2015, 85, 522-529. doi:10.1016/j.spmi.2015.04.035 
10. Ebrahimi, M.; Horri, A.; Sanaeepur, M.; Tavakoli, M. B. J. Appl. Phys. 2020, 127, 084504. doi:10.1063/1.5130777

11. Ebrahimi, M.; Horri, A.; Sanaeepur, M.; Tavakoli, M. B. J. Comput. Electron. 2020, 19, 62-69. doi:10.1007/s10825-019-01442-z

12. Pereira, J. M., Jr.; Vasilopoulos, P.; Peeters, F. M. Appl. Phys. Lett. 2007, 90, 132122. doi:10.1063/1.2717092

13. Nguyen, V. H.; Mazzamuto, F.; Bournel, A.; Dollfus, P. J. Phys. D: Appl. Phys. 2012, 45, 325104. doi:10.1088/0022-3727/45/32/325104

14. Song, Y.; Wu, H.-C.; Guo, Y. Appl. Phys. Lett. 2013, 102, 093118. doi:10.1063/1.4794952

15. Fallahazad, B.; Lee, K.; Kang, S.; Xue, J.; Larentis, S.; Corbet, C.; Kim, K.; Movva, H. C. P.; Taniguchi, T.; Watanabe, K.; Register, L. F.; Banerjee, S. K.; Tutuc, E. Nano Lett. 2015, 15, 428-433. doi:10.1021/nl503756y

16. Xiao, Z.; Ma, C.; Huang, J.; Liang, L.; Lu, W.; Hong, K.; Sumpter, B. G.; Li, A.-P.; Bernholc, J. Adv. Theory Simul. 2019, 2, 1800172. doi:10.1002/adts.201800172

17. Mazumder, P.; Kulkarni, S.; Bhattacharya, M.; Sun, J. P.; Haddad, G. I. Proc. IEEE 1998, 86, 664-686. doi:10.1109/5.663544

18. Pan, W.; Lyo, S. K.; Reno, J. L.; Simmons, J. A.; Li, D.; Brueck, S. R. J. Appl. Phys. Lett. 2008, 92, 052104. doi:10.1063/1.2840996

19. Hikita, Y.; Kourkoutis, L. F.; Susaki, T.; Muller, D. A.; Takagi, H.; Hwang, H. Y. Phys. Rev. B 2008, 77, 205330. doi:10.1103/physrevb.77.205330

20. Dalgleish, H.; Kirczenow, G. Nano Lett. 2006, 6, 1274-1278. doi:10.1021/nl060040m

21. Kovacic, S. J.; Ojha, J. J.; Simmons, J. G.; Jessop, P. E.; Mand, R. S.; SpringThorpe, A. J. IEEE Trans. Electron Devices 1993, 40, 1154-1160. doi:10.1109/16.214743

22. Seabaugh, A. C.; Luscombe, J. H.; Randall, J. N.; Colter, P. C.; Dip, A.; Eldallal, G. M.; Bedair, S. M. Thin Solid Films 1993, 225, 99-104. doi:10.1016/0040-6090(93)90135-c

23. Reed, M. A. Superlattices Microstruct. 1988, 4, 741-747. doi:10.1016/0749-6036(88)90206-6

24. Dubois, S. M.-M.; Declerck, X.; Charlier, J.-C.; Payne, M. C. ACS Nano 2013, 7, 4578-4585. doi:10.1021/nn401322t

25. Xu, Y.; Guo, Z.; Chen, H.; Yuan, Y.; Lou, J.; Lin, X.; Gao, H.; Chen, H.; Yu, B. Appl. Phys. Lett. 2011, 99, 133109. doi:10.1063/1.3643899

26. Li, M.; Zheng, B.; Duan, K.; Zhang, Y.; Huang, Z.; Zhou, H. J. Phys. Chem. C 2018, 122, 14945-14953. doi:10.1021/acs.jpcc.8b02750

27. Liu, X.; Zhang, G.; Zhang, Y.-W. Nano Lett. 2016, 16, 4954-4959. doi:10.1021/acs.nanolett.6b01565

28. Ding, N.; Lei, Y.; Chen, X.; Deng, Z.; Ng, S.-P.; Wu, C.-M. L. Comput. Mater. Sci. 2016, 117, 172-179. doi:10.1016/j.commatsci.2015.12.052

29. Zhang, J.; Xie, W.; Xu, X.; Zhang, S.; Zhao, J. Chem. Mater. 2016, 28, 5022-5028. doi:10.1021/acs.chemmater.6b01764

30. Pruneda, J. M. Phys. Rev. B 2012, 85, 045422. doi:10.1103/physrevb.85.045422

31. Li, Q.; Liu, M.; Zhang, Y.; Liu, Z. Small 2016, 12, 32-50. doi:10.1002/smll.201501766

32. Ding, N.; Chen, X.; Wu, C.-M. L. Sci. Rep. 2016, 6, 31499. doi:10.1038/srep31499

33. Wang, J.; Ma, F.; Liang, W.; Sun, M. Mater. Today Phys. 2017, 2, 6-34. doi:10.1016/j.mtphys.2017.07.001

34. Leon, C.; Costa, M.; Chico, L.; Latgé, A. Sci. Rep. 2019, 9, 3508. doi:10.1038/s41598-019-39763-5
35. Mukherjee, S.; Kaloni, T. P. J. Nanopart. Res. 2012, 14, 1059. doi:10.1007/s11051-012-1059-2

36. Sanaeepour, M.; Abedi, A.; Sharifi, M. J. IEEE Trans. Electron Devices 2017, 64, 1300-1304. doi:10.1109/ted.2017.2656944

37. Zhao, R.; Wang, J.; Yang, M.; Liu, Z.; Liu, Z. J. Phys. Chem. C 2012, 116, 21098-21103. doi:10.1021/jp306660x

38. Sanaeepur, M.; Goharrizi, A. Y.; Sharifi, M. J. IEEE Trans. Electron Devices 2014, 61, 1193-1198. doi:10.1109/ted.2013.2290049

39. Sancho, M. P. L.; Sancho, J. M. L.; Sancho, J. M. L.; Rubio, J. J. Phys. F: Met. Phys. 1985, 15, 851-858. doi:10.1088/0305-4608/15/4/009

40. Teong, H.; Lam, K.-T.; Liang, G. Jpn. J. Appl. Phys. 2009, 48, 04C156. doi:10.1143/jjap.48.04c156

41. Huang, B.; Liu, F.; Wu, J.; Gu, B.-L.; Duan, W. Phys. Rev. B 2008, 77, 153411. doi:10.1103/physrevb.77.153411

\section{License and Terms}

This is an Open Access article under the terms of the Creative Commons Attribution License (http://creativecommons.org/licenses/by/4.0). Please note that the reuse, redistribution and reproduction in particular requires that the authors and source are credited.

The license is subject to the Beilstein Journal of

Nanotechnology terms and conditions:

(https://www.beilstein-journals.org/bjnano)

The definitive version of this article is the electronic one which can be found at: doi:10.3762/bjnano.11.56 